greatly improved in every way. His memory, reasoning power, and articulation were almost perfect; there was no tremor of the lips or facial muscles, no tremor of the tongue nor overflow of saliva; yet there was the same evidence of spinal myosis, and the sharp lancinating pains continued to worry him. $\mathrm{He}$ had been taking large doses of the iodide of potassium with evident benefit. I need scarcely say that, in all human probability, this man will ere long become the subject of confirmed general paralysis, which was preceded by the symptoms of tabes about twelve years ago.

The following case, from a clinical and pathological point of view, is of exceptional interest, and shows how the signs of ataxy may exist, due to sclerosis of the posterior columns of the spinal cord in connection with pathological changes in the brain which are common to the general paralysis of the insane, without the patient presenting during life those objective psychical manifestations which usually are to be found in this disease. I do not assume that this case is in any way unique; but it is undoubtedly a typical connecting link in a certain chain of evidence -I mean the evidence of association between pathological changes in the brain and spinal cord of tabes on the one hand, and general paralysis of the insane on the other hand.

W. B., aged 34, was sent to consult me by Mr. Mackinlay, at the Hospital for Paralysis and Epilepsy, Regent's Park, in November I880. There was no family history of brain-disease, neither was there any clear evidence of syphilis. He had been employed as a dock-labourer, and enjoyed excellent health until the end of 1879 , when the left eyelid began to droop, and the sight of the left eye began to fail, and so did the sight of the right eye; and, after this, he saw double. In the month of July 1880, he began to suffer from severe headaches; the pain was of a shooting character, and extended from the forehead to the occiput, and sometimes from one temporal region to the other; and the pains would sometimes come on suddenly, and depart suddenly, but they were invariably associated with an increase of temperature. Soon after the pains in the head began, his gait became unsteady, and his fellow workmen accused him of being continually drunk. It is a point of interest to note that he never felt giddy during locomotion, but, when lying in bed, it seemed to him as though the bed were continually moving round and round; and, when sometimes standing upon his legs, his body revolved against his will from left to right. At times, he suffered from great stiffness of the muscles of the back of the neck, and the head would be forcibly retracted. The following notes of his actual state were made at the time of his admission into the hospital. "The cranial development is regular, and not unduly prominent in any direction. When the skull is percussed, he experiences no pain, but, some months ago, the scalp was so tender, that he could not bear it to be touched. I am indebted to Mr. Mackinlay for the following ophthalmoscopic examination. Right eye : discs white (a little bluish); punched out in appearance; no cupping; arteries very small indeed (like fine hairs); veins about normal size; choroid thin; tension normal; vision equal $\frac{2}{7} \frac{\mathrm{g}}{8}$ partly, and Jäger 4 at 8 inches (minim type). Left eye : disc dead white and punched out: veins tortuous, arteries very small indeed; tension normal ; vision absolutely nil. The pupils, moderate in size, rather larger in the right eye than in the left, do not respond, even to very strong light. Slight ptosis of left lid. There are no marked objective signs of any other cranial nerves being involved. He complains of numbness at the tips of the fingers, but there are no other subjective symptoms of defective sensation. On the objective side, there is a marked retardation in the conduction of impressions from the periphery to the nervous centres and more especially in the lower limbs, both in reference to pain and sensation. All voluntary movements are co-ordinate when he is lying down, but, when standing or walking, there is defective volitional co-ordinating power. There is complete absence of knee-reflex, and no reflex movements are excited when the soles of the feet are tickled. The sphincter muscles of the bladder and of the rectum are unaffected. There is a marked tendency to depression, and he is readily induced to cry and to laugh. Sometimes (but not often), there is defect of articulation otherwise, there is neither tremor of the tongue nor of the facial muscles.'

These signs and symptoms deserve consideration; for I maintain now, as I maintained then, that we had clear evidence of gross intracranial change, which involved the cerebellum; and I am quite willing to admit with all candour, that I even thought my patient had a tumour of the cerebellum. Dr. Buzzard, who saw the case, thought there was no question of doubt about its being a case of tabes, pure and simple; and my friend Dr. Boyd, with his vast experience and great mental acumen in all such matters, suggested "general paralysis of the insane". In about three months from this time, the man died, with no other marked signs or symptoms than those just narrated, excepting the signs of a more or less acute general paralysis, into the details of which it is not necessary to enter.
At the post mortem examination, which was kindly made for me by my then colleague Dr. Sturge, the following notes were made. The dura mater was much thickened, especially over the vertex and the anterior lobes. There was much meningeal congestion and diffuse chronic arachnitis, with considerable excess of fluid; adhesions existed between the membranes and the cortex. The convolutions were particularly noticeable over the frontal gyri, and the gyri of the temporo-sphenoidal lobes, as well as the ascending parietal convolutions. The grey cortex was materially altered and wasted and softened in the parts just mentioned, and the white substance of the hemispheres was excessively vascular. At the base of the brain, marked changes had taken place, but more particularly was this the case in the optic nerves; they were shrivelled and hardened, and looked something like dried gelatine; this was seen more clearly towards their ends, for, where they wound round the thalamus, the structures appeared to be normal. There was the same condition of arachnitis at the base that there was over the vertex of the brain. The cerebellum was healthy, but there were some adhesions of a slight character over its surface. The floor of the fourth ventricle was remarkably granular; and I may here mention that this condition is common in the brain of the general paralytic. The spinal cord presented slight chronic inflammatory thickening of the membranes, but no adhesions. I am indebted to my friend Dr. W. B. Kesteven for the following careful microscopic examination. Beginning at the conus medullaris, and going up through the spinal cord to the cervical region, there was marked sclerosis of the posterior columns, extending slightly into the lateral columns. Between the posterior cornua, the columns had a darker aspect than in healthy cords, owing to an in. crease of the connective tissue and its fibrillation, which encroached upon the nerve-fibres, compressing them and their axis-cylinders, so that, in a transverse section of these portions, the translucent sheath and coloured axis-cylinders presented an opaque aspect. These portions of the cord took dges more deeply than the healthy parts. The texture of the cornua also was much denser than in the normal state. Their cells had not undergone any alteration of size, but in many places they were slightly granular, and their nuclei appeared to me enlarged. The cells of the anterior horns were distinctly undergoing fuscous degeneration in varying degrees, as shown by their only partially taking the carmine dye. The roots of the nerves were, in many instances, to some extent atrophied. The blood-vessels were tortuous, and had become dilated by repeated distension, so that large perivascular spaces were seen in which the cut ends of vessels were observed to be thickened. In many of the smaller vessels were thrombi and emboli. These lesions were most obvious in the cervical region and in the medulla oblongata. The appearance of sclerosis decreased upwards, until it had disappeared in the medulla, where, as in the anterior horns of the cervical region, the cells seemed to be the principal seat of the morbid changes, very marked in those of the olivary bodies, and in the nuclei of the vagus. In the medulla oblongata were scattered a few colloid bodies. The central canal throughout its whole length was obliterated by nuclei and corpuscles. The fibres of the optic nerve and commissures were very much atrophied, whilst their blood-vessels were enlarged and much dilated.

Such, then, are the notes, clinical and pathological, of a case which, I hold, presents features common alike to the general paralytic and tabetic. I have no time here to analyse these especial features, but, in the following remarks, the drift of my ideas will be elucidated.

( $T o$ be continued.)

\section{NOTES OF A CASE OF ACUTE ASCENDING} PARALYSIS.

Bx J. MAGEE FINNY, M.D.Dubl., F.K.Q.C.P.I., Visiting Physician to, and Lecturer on Clinical Medicine in, the City of Dublin Hospital.

THIS disease is one of very great rarity. In 1859, Dr. Landry described a peculiar form of paralysis, which he termed "Paralysie ascendante aiguë" (Gaz. Hcbdom., 1859, Nos. 30, 31); and yet not more than thirty cases are to be found recorded, and some even of these are doubtful examples of that disease. This lack of instances is the more noticeable, since the last decade is one marked in the history of medicine for the very great interest taken, and the rapid advances made, by many skilful observers in diseases of the nervous system.

Of the etiology and pathology of acute ascending paralysis, it may be truly said we have no knowledge. It seems to have no morbid ana. tomy. The most careful and experienced observers-such as Vulpian, Hayem, Westphal, Pelligrino, [Levi, and MM. Déjérine and Goetz- 
have failed to discover any gross or microscopical lesion, or any anatomical changes (which could in any way be connected with the production of the symptoms peculiar to Landry's disease), with reference to the brain, medulla, and spinal cord ; or to the sympathetic nerve, the peripheral nerve-trunks, or the muscles. (Diseases of the Spinal Cord, by Erb : Ziemssen's Encyclopadia, vol. xiii.) On the other hand, it should be stated that Drs. Harley and Lockhart Clarke noted distinct degenerative changes, in parts of the spinal cord, in a case of acute progressive paralysis described by them (Lancel, 1868, October 3rd); and, in his book on Diseases of the Nervous System, published in 188r, Dr. Ross records pathological alterations in the grey nucleus of the cord, in a supposed case of this disease-a case, however, which he had not seen during life. With these exceptions, and perhaps that in which MM. Déjérine and Goetz discovered atrophy of some tubuli, with an increase of nuclei of the intertubular connective tissue in a single nerve root, I know of no published instances of the disease in which anything ab. normal has been discovered in the nervous centres, which might be termed peculiar.

The absence of a distinct pathology suggested, doubtless, to Landry the idea that acute ascending paralysis is not a disease of the spinal cord, but is due to some intoxication, which evidences its pernicious influence through the motor tracts of nervous centres by some inappreciable physico-chemical change in the nerve-elements. This view, which, for want of a better, was adopted by Westphal, seems to gather some additional weight from the close analogy, which Dr. Harley has noted, between the symptoms of the disease and the physiological effects of woorara on animals.

On this question, it is plainly premature to give an opinion; and one must be content to look upon its pathology and etiology through the same misty cloud of ignorance as that through which we view the pathology of chorea, tetanus, hydrophobia, and some other affections which are characterised by strong perversion of nervous function.

The following case of acute ascending paralysis came under my observation in December 1879 , and constitutes the only instance I have met; and, as far as I know, the only recognised instance of the disease which has ever occurred in Dublin. Although some of the details of the case are, I regret to say, lacking in that accuracy which I could desire, and which a more intimate knowledge, at the time, of the nature and main features of the disease would have enabled me to have supplied, I consider the following notes of the case worthy of notice.

CASE. - Helen M., aged 25, was admitted to the City of Dublin Hospital, under my care, on December $8 \mathrm{th}, 1879$, complaining of great weakness of her legs, and of inability to walk or stand. When seen on the 9 th, her aspect and position in bed presented nothing to warrant the idea that, within five days, she would have passed away. Her decubitus was dorsal, but easy, the legs lying extended in the natural position of repose. With great efforts, she was able to sit up; but she could not turn on her side without the nurse's assistance. The legs lay motionless, and she was unable to stir them or bend them in the least. There was a total absence of all rigidity or spasm on passive movement, or of fibrillary twitchings. On testing the museles of the thigh and leg by faradism, the electro-muscular contractility and sensibility seemed normal, or but little diminished. Sensation was unimpaired, and responded to the tests of touching, pricking, and of heat and cold. The superficial reflexes were not exaggerated. Examination of the spine failed to elicit any marked point of tenderness to firm pressure, percussion, or the hot sponge. The patient did not complain of the sensation of $a$ belt around the waist, or of undue heat or cold-symptoms with which one is familiar in some cases of inflammatory affections of the spinal cord-though she referred to an uncomfortable feeling above the sacrum; and this, I take it, was but the expression of powerlessness of the parts below that point. There was no paralysis of the bladder or rectum during the first two days of her stay in hospital, and the urine was of normal reaction and specific gravity, and free from albumen. There were no bed-sores, and the nutrition of the limbs was unimpaired, the muscles being of equal size in each leg.

Thinking it possible that much of the symptoms could be explained on the theory of the case being one of hysteria - an hypothesis strongly suggested by the patient's emotional manner, and familiar and endearing mode of speech-I had her lifted out of bed by two assistants, for the purpose of testing her powers of standing and of locomotion. It was at once evident that she was totally unable to direct her legs in the very slightest degree, still less to stand or to walk. Her legs hung quite powerless, and, as if loose at the hips, they dragged after her as she was lifted out of bed and put back again, without the least attempt at spasm or rigidity. In fact, they reminded one forcibly of the limbs one sees in advanced infantile palsy.

There seemed on the patient's part every readiness to make the best effort in her power; and, although she was agitated by being lifted out of bed, she offered no resistance. It was now plain that a very serious lesion of the motor tracts of the spinal cord existed; or, speaking more correctly, a serious disturbance of motility was present; and that the disease had advanced with great rapidity during the preceding four days-seeing that she had lost, during that time, all power of motion in the lower extremities and in the back.

The next two days evidenced still further progress of the disease upwards, as she lost-at first partially, and then completely-the power over the arms and hands. This was shown by her inability to draw the bedclothes up, or to arrange her night-dress, or to lift herself up in the bed. The spinal muscles also failed, and it required constant care on the part of the nurse to keep her propped up in bed. She could not lie down, owing to oppression of breathing.

On December 12th, the respiration was almost entirely carried on by the diaphragm. On the $13^{\text {th }}$, the paralysis involved the powers of deglutition; the pulse ran up to 136 ; and she sank on the 14 th, with all the symptoms of asphyxia-the mind remaining clear till about fifteen hours before the close.

After the third day of her stay in hospital, it became necessary to draw off the urine, and the bowels became constipated-a condition due largely to the paralysed state of the abdominal muscles. The arine was at no time ammoniacal.

In seeking for evidence to throw some light upon this novel case, I made careful inquiries into her history. From herself, little could be learned, as she was completely silent as to her immediate antecedents; and, though her sisters were able to supply some facts, there was nothing, I regret to say, discernible, which could have acted as a predisponent or excitant of the disease, or which threw any light upon its nature. Though unmarried, it appeared she kept company occasionally for over a year with a man, who seemed to evince kindness towards her, and at whose hands she apparently received no ill-treatment. About a fortnight or three weeks before her present illness, she left her home in this man's company, and he helped her back three days before admission into hospital. On that occasion, her sisters stated, she was just able to stand, and, by the use of her arms, to drag her legs after her into the hall, but was unable to go upstairs. She remained at home three days; and, during them, she lost all power of moving her feet and legs. Though questioned closely by her sisters, she gave them no insight into the origin of her illness-if, indeed, it were in her power to do so. There were no appearances to warrant the idea of her having taken too much drink, or of having received any ill-treatment or hurt. When seen by me, there were no marks of blows or violence on any part of her body. She was well-nourished and well-formed, with fair muscular development, and without any evidence of either scrofula or syphilis.

The duration of the disease was somewhere between nine and twelve days, as it is prcbable she was complaining of some degree of loss of power before she returned home, three days before admission to hos. pital, and nine before death.

The indications for treatment were by no means clear, so that I directed my attention to supporting her general strength by light nutritious diet, and to giving her sleep, and to calming her emotional excitement. For this purpose, I gave her bromide of potassium, in fifteen-grain doses, three times a day, and sleeping draughts of chloral. The insomnia, however, was not readily overcome, as she needed rather large doses of morphia (hypodermically administered) before sleep was procured.

At the post mortem examination, which I conducted, with the assistance of my clinical clerk (Dr. Stuart Davis), twenty-four hours after death, there was noted nothing abnormal in the thoracic or abdominal cavities, with the exception of a cyst, of the size of a walnut, in the left ovary. The brain and spinal cord were carefully removed. Sections were made through several parts of both, but I could discover no gross or macroscopical alterations of the nervous tissue, or in its coverings, which could be termed peculiar, or which could not be accounted for by the mode of death of the patient. As to the microscopical appearances presented by the spinal cord, I regret to say I am unable to speak, as, by a most lamentable oversight, the specimens were not placed in hardening fuid till decomposition had set in, and rendered them use less for microscopical examination. As, however, but negative results have followed most elaborate and careful microscopical examinations made of the nervous centres (by more skilled hands than mine), this was not a matter of so much regret as it would otherwise have been.

In conclusion, from a careful review of the whole case, its gradual onset, its steady but rapid upward progression, its fatal termination by involving respiration and deglutition, and the absence of all irritation of the spinal cord or its coverings, there can be no doubt of its having been a typical example of Landry's disease, occurring in a woman. 